\title{
Weak limit of iterates of some random-valued functions and its application
}

\author{
KAROL BAROND
}

Dedicated to Professor János Aczél on his 95th birthday.

Abstract. Given a probability space $(\Omega, \mathcal{A}, P)$, a complete and separable metric space $X$ with the $\sigma$-algebra $\mathcal{B}$ of all its Borel subsets, a $\mathcal{B} \otimes \mathcal{A}$-measurable and contractive in mean $f: X \times \Omega \rightarrow X$, and a Lipschitz $F$ mapping $X$ into a separable Banach space $Y$ we characterize the solvability of the equation

$$
\varphi(x)=\int_{\Omega} \varphi(f(x, \omega)) P(d \omega)+F(x)
$$

in the class of Lipschitz functions $\varphi: X \rightarrow Y$ with the aid of the weak limit $\pi^{f}$ of the sequence of iterates $\left(f^{n}(x, \cdot)\right)_{n \in \mathbb{N}}$ of $f$, defined on $X \times \Omega^{\mathbb{N}}$ by $f^{0}(x, \omega)=x$ and $f^{n}(x, \omega)=$ $f\left(f^{n-1}(x, \omega), \omega_{n}\right)$ for $n \in \mathbb{N}$, and propose a characterization of $\pi^{f}$ for some special rvfunctions in Hilbert spaces.

Mathematics Subject Classification. Primary 39B12, 26A18; Secondary 60B12, 58D20.

Keywords. Random-valued functions, Iterates, Weak limit, Iterative equations, Lipschitzian solutions, Bochner integral, Gaussian measures.

\section{Introduction}

Fix a probability space $(\Omega, \mathcal{A}, P)$ and a complete and separable metric space $(X, \rho)$.

Let $\mathcal{B}$ denote the $\sigma$-algebra of all Borel subsets of $X$. We say that $f$ : $X \times \Omega \rightarrow X$ is a random-valued function (shortly: an rv-function) if it is measurable with respect to the product $\sigma$-algebra $\mathcal{B} \otimes \mathcal{A}$. The iterates of such an rv-function are given by

$$
f^{0}\left(x, \omega_{1}, \omega_{2}, \ldots\right)=x, \quad f^{n}\left(x, \omega_{1}, \omega_{2}, \ldots\right)=f\left(f^{n-1}\left(x, \omega_{1}, \omega_{2}, \ldots\right), \omega_{n}\right)
$$


for $n \in \mathbb{N}, x \in X$ and $\left(\omega_{1}, \omega_{2}, \ldots\right)$ from $\Omega^{\infty}$ defined as $\Omega^{\mathbb{N}}$. Note that $f^{n}: X \times$ $\Omega^{\infty} \rightarrow X$ is an rv-function on the product probability space $\left(\Omega^{\infty}, \mathcal{A}^{\infty}, P^{\infty}\right)$. More exactly, for $n \in \mathbb{N}$ the $n$th iterate $f^{n}$ is $\mathcal{B} \otimes \mathcal{A}_{n}$-measurable, where $\mathcal{A}_{n}$ denotes the $\sigma$-algebra of all sets of the form

$$
\left\{\left(\omega_{1}, \omega_{2}, \ldots\right) \in \Omega^{\infty}:\left(\omega_{1}, \ldots, \omega_{n}\right) \in A\right\}
$$

with $A$ from the product $\sigma$-algebra $\mathcal{A}^{n}$. (See [8, Sec. 1.4], [6].)

Let $f: X \times \Omega \rightarrow X$ be an rv-function.

A result on the a.s. convergence of $\left(f^{n}(x, \cdot)\right)_{n \in \mathbb{N}}$ for $X$ being the unit interval may be found in [8, Sec. 1.4B]. The paper [6] by Rafał Kapica brings theorems on the convergence a.s. and in $L^{1}$ of those sequences of iterates in the case where $X$ is a closed subset of a Banach lattice. A simple criterion for the convergence in law of $\left(f^{n}(x, \cdot)\right)_{n \in \mathbb{N}}$ to a random variable independent of $x \in X$ was proved in [1] and applied to the equation

$$
\varphi(x)=\int_{\Omega} \varphi(f(x, \omega)) P(d \omega)+F(x)
$$

with $\varphi$ as the unknown function. This criterion reads as follows.

(H) There exists a $\lambda \in(0,1)$ such that

$$
\int_{\Omega} \varrho(f(x, \omega), f(z, \omega)) P(d \omega) \leq \lambda \varrho(x, z) \quad \text { for } x, z \in X
$$

and

$$
\int_{\Omega} \varrho(f(x, \omega), x) P(d \omega)<\infty \quad \text { for } x \in X .
$$

Thus, denoting by $\pi_{n}^{f}(x, \cdot)$ the distribution of $f^{n}(x, \cdot)$, i.e.,

$$
\pi_{n}^{f}(x, B)=P^{\infty}\left(f^{n}(x, \cdot) \in B\right) \quad \text { for } n \in \mathbb{N} \cup\{0\}, x \in X \text { and } B \in \mathcal{B},
$$

hypothesis $(\mathrm{H})$ guarantees the existence of a probability Borel measure $\pi^{f}$ on $X$ such that

$$
\lim _{n \rightarrow \infty} \int_{X} u(z) \pi_{n}^{f}(x, d z)=\int_{X} u(z) \pi^{f}(d z)
$$

holds for $x \in X$ and for any continuous and bounded $u: X \rightarrow \mathbb{R}$; more exactly, cf. also [2, Theorem 3.1],

$$
\int_{X} \varrho(x, z) \pi^{f}(d z)<\infty \quad \text { for } x \in X
$$

and

$$
\left|\int_{X} u(z) \pi_{n}^{f}(x, d z)-\int_{X} u(z) \pi^{f}(d z)\right| \leq \frac{\lambda^{n}}{1-\lambda} \int_{X} \varrho(f(x, \omega), x) P(d \omega)
$$

for $x \in X, n \in \mathbb{N}$ and a non-expansive $u$ mapping $X$ into $[-1,1]$.

Rafał Kapica strengthened this estimation showing, see [7, Corollary 5.6 and Lemma 3.1], that (5) holds for $x \in X, n \in \mathbb{N}$ and a non-expansive $u$ : $X \rightarrow \mathbb{R}$. Since it is explicitly stated there only for a non-expansive and bounded 
$u: X \rightarrow \mathbb{R}$ we prove it for a non-expansive $u$ mapping $X$ into a separable Banach space making use of (5) for non-expansive and bounded $u: X \rightarrow \mathbb{R}$ only. Having done that we characterize the solvability of (1) in the class of Lipschitz functions with the aid of this limit distribution $\pi^{f}$. Moreover, we propose a characterization of $\pi^{f}$ for some special rv-functions in Hilbert spaces.

\section{Solvability of the equation}

Following [3] given an rv-function $f: X \times \Omega \rightarrow X$ such that $(\mathrm{H})$ holds and a Lipschitz $F$ mapping $X$ into a separable Banach space $Y$ define

$$
F_{0}(x)=F(x), \quad F_{n}(x)=\int_{\Omega} F_{n-1}(f(x, \omega)) P(d \omega)
$$

for $x \in X$ and $n \in \mathbb{N}$, and note that according to [3, Theorem 2.1] there exists a $y_{0} \in Y$ such that for every $x \in X$ the sequence $\left(F_{n}(x)\right)_{n \in \mathbb{N}}$ converges to $y_{0}$, and any Lipschitz solution $\varphi: X \rightarrow Y$ of (1) has the form

$$
\varphi(x)=c+\sum_{n=0}^{\infty} F_{n}(x) \text { for } x \in X
$$

where $c$ is a constant from $Y$. With the aid of the limit distribution $\pi^{f}$ we characterize this pointwise limit of $\left(F_{n}\right)_{n \in \mathbb{N}}$ and, making use of $[3$, Theorem 2.1(ii)], the solvability of (1) as follows (cf. [1, Corollary 4.1]).

Theorem 2.1. Assume $(\mathrm{H})$. If $F$ is a Lipschitz mapping of $X$ into a separable Banach space $Y$, then

$$
\lim _{n \rightarrow \infty} F_{n}(x)=\int_{X} F(z) \pi^{f}(d z) \quad \text { for } x \in X
$$

and Eq. (1) has a Lipschitz solution $\varphi: X \rightarrow Y$ if and only if

$$
\int_{X} F(z) \pi^{f}(d z)=0
$$

As announced above, we start with the following lemma.

Lemma 2.2. If $f: X \times \Omega \rightarrow X$ is an rv-function such that (2) holds with a $\lambda \in(0,1)$ and $(3)$ is satisfied, then

$$
\left\|\int_{X} u(z) \pi_{n}^{f}(x, d z)-\int_{X} u(z) \pi^{f}(d z)\right\| \leq \frac{\lambda^{n}}{1-\lambda} \int_{X} \varrho(f(x, \omega), x) P(d \omega)
$$

for $x \in X, n \in \mathbb{N}$ and for any non-expansive $u$ mapping $X$ into a separable Banach space. 
Proof. First of all let us observe that for every $x \in X, n \in \mathbb{N}$ and $\left(\omega_{1}, \omega_{2}, \ldots\right) \in$ $\Omega^{\infty}$ we have

$$
\begin{aligned}
& \rho\left(f^{n}\left(x, \omega_{1}, \omega_{2}, \ldots\right), x\right)=\rho\left(f^{n-1}\left(f\left(x, \omega_{1}\right), \omega_{2}, \omega_{3}, \ldots\right), x\right) \\
& \quad \leq \sum_{k=1}^{n} \rho\left(f^{n-k}\left(f\left(x, \omega_{k}\right), \omega_{k+1}, \omega_{k+2}, \ldots\right), f^{n-k}\left(x, \omega_{k+1}, \omega_{k+2}, \ldots\right)\right),
\end{aligned}
$$

where the value $f^{n}\left(x, \omega_{1}, \omega_{2}, \ldots\right)$ depends only on $x$ and $\left(\omega_{1}, \ldots, \omega_{n}\right)$, and by (2) we have

$$
\int_{\Omega^{\infty}} \varrho\left(f^{n}(x, \omega), f^{n}(z, \omega)\right) P^{\infty}(d \omega) \leq \lambda^{n} \varrho(x, z) \quad \text { for } x, z \in X \text { and } n \in \mathbb{N} .
$$

Hence, applying the Fubini theorem, for $x \in X$ and $n \in \mathbb{N}$ we get

$$
\begin{aligned}
& \int_{X} \rho(x, z) \pi_{n}^{f}(x, d z)=\int_{\Omega^{\infty}} \rho\left(f^{n}(x, \omega), x\right) P^{\infty}(d \omega) \\
& \quad \leq \sum_{k=1}^{n} \int_{\Omega^{\infty}} \rho\left(f^{n-k}\left(f\left(x, \omega_{1}\right), \omega_{2}, \omega_{3}, \ldots\right), f^{n-k}\left(x, \omega_{2}, \omega_{3}, \ldots\right)\right) P^{\infty}\left(d\left(\omega_{1}, \omega_{2}, \ldots\right)\right) \\
& \quad \leq \sum_{k=1}^{n} \lambda^{n-k} \int_{\Omega} \rho(f(x, \omega), x) P(d \omega) \leq \frac{1}{1-\lambda} \int_{\Omega} \varrho(f(x, \omega), x) P(d \omega) .
\end{aligned}
$$

Consequently, for any non-expansive $u$ mapping $X$ into a separable Banach space and for every $x \in X, n \in \mathbb{N}$ we obtain

$$
\int_{X}\|u(z)\| \pi_{n}^{f}(x, d z) \leq \frac{1}{1-\lambda} \int_{\Omega} \varrho(f(x, \omega), x) P(d \omega)+\|u(x)\| ;
$$

moreover,

$$
\int_{X}\|u(z)\| \pi^{f}(d z) \leq \int_{X} \varrho(x, z) \pi^{f}(d z)+\|u(x)\| .
$$

Let $u$ be a non-expansive mapping of $X$ into a separable Banach space $Y$. To show that (8) holds for $x \in X$ and $n \in \mathbb{N}$ we may assume that $Y$ is a real space.

Fix $x \in X, n \in \mathbb{N}$ and then a $y^{*} \in Y^{*}$ such that $\left\|y^{*}\right\| \leq 1$ and

$$
\left\|\int_{X} u(z) \pi_{n}^{f}(x, d z)-\int_{X} u d \pi^{f}\right\|=y^{*}\left(\int_{X} u(z) \pi_{n}^{f}(x, d z)-\int_{X} u d \pi^{f}\right) .
$$

For every $k \in \mathbb{N}$ the function $\tau_{k}: \mathbb{R} \rightarrow \mathbb{R}$ given by $\tau_{k}(t)=-k$ for $t \in(-\infty,-k)$, $\tau_{k}(t)=t$ for $t \in[-k, k], \tau_{k}(t)=k$ for $t \in(k, \infty)$ is non-expansive and $\left|\tau_{k}(t)\right| \leq|t|$ for $t \in \mathbb{R}$. Consequently, since (5) holds for every non-expansive and bounded $u: X \rightarrow \mathbb{R}$, for every $k \in \mathbb{N}$ we have

$$
\begin{aligned}
& \left|\int_{X} \tau_{k}\left(y^{*} u(z)\right) \pi_{n}^{f}(x, d z)-\int_{X} \tau_{k}\left(y^{*} u(z)\right) \pi^{f}(d z)\right| \\
& \quad \leq \frac{\lambda^{n}}{1-\lambda} \int_{X} \varrho(f(x, \omega), x) P(d \omega)
\end{aligned}
$$


and, by (9) and (10),

$$
\begin{aligned}
& \int_{X}\left|\tau_{k}\left(y^{*} u(z)\right)\right| \pi_{n}^{f}(x, d z) \leq \frac{1}{1-\lambda} \int_{\Omega} \varrho(f(x, \omega), x) P(d \omega)+\|u(x)\|, \\
& \int_{X}\left|\tau_{k}\left(y^{*} u(z)\right)\right| \pi^{f}(d z) \leq \int_{X} \varrho(x, z) \pi^{f}(d z)+\|u(x)\| .
\end{aligned}
$$

Hence, taking (3) and (4) into account, applying the Lebesgue dominated convergence theorem and passing with $k$ to the limit in (12) we get

$$
\begin{aligned}
& \left|\int_{X} y^{*} u(z) \pi_{n}^{f}(x, d z)-\int_{X} y^{*} u(z) \pi^{f}(d z)\right| \\
& \quad \leq \frac{\lambda^{n}}{1-\lambda} \int_{X} \varrho(f(x, \omega), x) P(d \omega)
\end{aligned}
$$

and (8) follows now from (11).

Proof of the theorem. An easy induction shows that

$$
F_{n}(x)=\int_{\Omega^{\infty}} F\left(f^{n}(x, \omega)\right) P^{\infty}(d \omega),
$$

i.e.,

$$
F_{n}(x)=\int_{X} F(z) \pi_{n}^{f}(x, d z) \quad \text { for } x \in X, n \in \mathbb{N} .
$$

Let $L$ be a Lipschitz constant for $F$. Putting $u=\frac{1}{L} F$ we have (8), whence

$$
\left\|F_{n}(x)-\int_{X} F(z) \pi^{f}(d z)\right\| \leq \frac{L \lambda^{n}}{1-\lambda} \int_{X} \varrho(f(x, \omega), x) P(d \omega)
$$

for $x \in X$ and $n \in \mathbb{N}$. It proves (6) and according to [3, Theorem 2.1(ii)] Eq. (1) has a Lipschitz solution $\varphi: X \rightarrow Y$ if and only if (7) holds.

\section{A characterization of the limit distribution}

Obviously the problem of characterization of the limit distribution $\pi^{f}$ arises. The following theorem provides a characterization via functional equations for some special rv-functions in Hilbert spaces. More exactly, we characterize $\pi^{f}$ via a functional equation for its characteristic function $\varphi^{f}: X \rightarrow \mathbb{C}$,

$$
\varphi^{f}(u)=\int_{X} e^{i(u \mid z)} \pi^{f}(d z),
$$

cf. [10] by O. K. Zakusilo. Note that any two probability Borel measures on $X$ with the same characteristic function are equal, see [9, Ch. VI, Th. 2.1(2)]. 
Theorem 3.1. Assume $X$ is a real separable Hilbert space, $\Lambda: X \rightarrow X$ is linear and continuous, $\xi: \Omega \rightarrow X$ is $\mathcal{A}$-measurable, and

$$
f(x, \omega)=\Lambda x+\xi(\omega) \quad \text { for }(x, \omega) \in X \times \Omega .
$$

If

$$
\|\Lambda\|<1 \quad \text { and } \quad \int_{\Omega}\|\xi(\omega)\| P(d \omega)<\infty,
$$

then the characteristic function of $\pi^{f}$ is the only solution $\varphi: X \rightarrow \mathbb{C}$ of the equation

$$
\varphi(u)=\gamma(u) \varphi\left(\Lambda^{*} u\right)
$$

which is continuous at zero and fulfils $\varphi(0)=1$, where $\gamma$ stands for the characteristic function of $\xi$.

Proof. For $n \in \mathbb{N}$ define $\xi_{n}: \Omega^{\infty} \rightarrow X$ by $\xi_{n}\left(\omega_{1}, \omega_{2}, \ldots\right)=\xi\left(\omega_{n}\right)$ and note that $\xi_{n}, n \in \mathbb{N}$, are identically distributed: Denoting by $\rho$ the distribution of $\xi$ we have

$$
P^{\infty}\left(\xi_{n} \in B\right)=P(\xi \in B)=\rho(B)
$$

for $n \in \mathbb{N}$ and $B \in \mathcal{B}$. Since

$$
f^{n}(x, \omega)=\Lambda f^{n-1}(x, \omega)+\xi_{n}(\omega) \text { for } \omega \in \Omega^{\infty},
$$

and the random variables $\Lambda \circ f^{n-1}(x, \cdot), \xi_{n}$ are independent, we see that

$$
\pi_{n}^{f}(x, \cdot)=\left(\pi_{n-1}^{f}(x, \cdot) \circ \Lambda^{-1}\right) * \rho \quad \text { for } n \in \mathbb{N}, x \in X .
$$

Hence, passing to the limit (cf. [9, Ch. III, Th. 1.1]),

$$
\pi^{f}=\left(\pi^{f} \circ \Lambda^{-1}\right) * \rho .
$$

Consequently, see also [9, p. 58], for $u \in X$,

$$
\begin{aligned}
\varphi^{f}(u) & =\int_{X} e^{i(u \mid z)}\left(\left(\pi^{f} \circ \Lambda^{-1}\right) * \rho\right)(d z) \\
& =\int_{X \times X} e^{i(u \mid x+y)}\left(\left(\pi^{f} \circ \Lambda^{-1}\right) \times \rho\right)(d(x, y)) \\
& =\int_{X}\left(\int_{X} e^{i(u \mid x)} \cdot e^{i(u \mid y)}\left(\pi^{f} \circ \Lambda^{-1}\right)(d x)\right) \rho(d y) \\
& =\left(\int_{X} e^{i(u \mid x)}\left(\pi^{f} \circ \Lambda^{-1}\right)(d x)\right)\left(\int_{X} e^{i(u \mid y)} \rho(d y)\right) \\
& =\left(\int_{X} e^{i(u \mid \Lambda x)} \pi^{f}(d x)\right) \gamma(u)=\varphi^{f}\left(\Lambda^{*} u\right) \gamma(u) .
\end{aligned}
$$


To prove the uniqueness consider a continuous at zero solution $\varphi: X \rightarrow \mathbb{C}$ of (13) such that $\varphi(0)=1$. Then

$$
\varphi(u)=\varphi\left(\left(\Lambda^{*}\right)^{n} u\right) \prod_{k=0}^{n-1} \gamma\left(\left(\Lambda^{*}\right)^{k} u\right) \quad \text { for } n \in \mathbb{N}, u \in X,
$$

and $\lim _{n \rightarrow \infty}\left(\Lambda^{*}\right)^{n} u=0$ for $u \in X$, whence

$$
\varphi(u)=\prod_{n=0}^{\infty} \gamma\left(\left(\Lambda^{*}\right)^{n} u\right) \quad \text { for } u \in X .
$$

\section{Examples}

1. Fix an integer $n \geq 2$ and a Lipschitz mapping $F$ of $\mathbb{R}$ into a separable Banach space $Y$ and consider the equation

$$
\varphi(x)=\frac{1}{n} \sum_{k=0}^{n-1} \varphi\left(\frac{x+k}{n}\right)+F(x) .
$$

In this case

$$
f(x, \omega)=\frac{1}{n} x+\frac{\omega}{n} \quad \text { for } x \in \mathbb{R}, \omega \in\{0,1, \ldots n-1\},
$$

$\xi(\omega)=\frac{\omega}{n}$ and $P(\{\omega\})=\frac{1}{n}$ for $\omega \in\{0,1, \ldots n-1\}$. Hence the characteristic function $\gamma$ of $\xi$ is given by

$$
\gamma(u)=\frac{1}{n} \sum_{k=0}^{n-1} \exp \left(i u \frac{k}{n}\right) \quad \text { for } u \in \mathbb{R} .
$$

A simple calculation shows that the characteristic function of the uniform distribution $U(0,1)$, i.e. the function $\phi: \mathbb{R} \rightarrow \mathbb{C}$ given by

$$
\phi(u)=\frac{e^{i u}-1}{i u} \quad \text { for } u \neq 0, \quad \phi(0)=1,
$$

satisfies

$$
\phi(u)=\gamma(u) \phi\left(\frac{1}{n} u\right) \quad \text { for } u \in \mathbb{R} .
$$

Hence and from Theorem 3.1 we infer that $\pi^{f}=U(0,1)$, i.e., $\pi^{f}(B)=$ $\lambda_{1}(B \cap[0,1])$ for Borel $B \subset \mathbb{R}$, where $\lambda_{1}$ denotes the one-dimensional Lebesgue measure. Applying now Theorem 2.1 we see that Eq. (14) has a Lipschitz solution $\varphi: \mathbb{R} \rightarrow Y$ if and only if 


$$
\int_{[0,1]} F(z) d z=0
$$

Cf. [3, Example 2.2].

2. Fix an $\alpha \in(-1,1)$ and a Lipschitz mapping $F$ of $\mathbb{R}$ into a separable Banach space $Y$ and consider the equation

$$
\varphi(x)=\int_{\Omega} \varphi(\alpha x+\xi(\omega)) P(d \omega)+F(x)
$$

assuming that $\xi: \Omega \rightarrow \mathbb{R}$ is a random variable with the Gaussian law $N\left(m, \sigma^{2}\right)$, i.e. the equation

$$
\varphi(x)=\frac{1}{\sigma \sqrt{2 \pi}} \int_{\mathbb{R}} \varphi(\alpha x+y) e^{-\frac{(y-m)^{2}}{2 \sigma^{2}}} d y+F(x),
$$

where $m$ is a real number and $\sigma$ is a positive real number. In this case

$$
\begin{aligned}
& f(x, \omega)=\alpha x+\xi(\omega) \quad \text { for }(x, \omega) \in \mathbb{R} \times \Omega, \\
& \gamma(u)=\exp \left(i m u-\frac{1}{2} \sigma^{2} u^{2}\right) \quad \text { for } u \in \mathbb{R},
\end{aligned}
$$

and, as a simple calculation shows, the function $\phi: \mathbb{R} \rightarrow \mathbb{C}$ given by

$$
\phi(u)=\exp \left(i \frac{m}{1-\alpha} u-\frac{1}{2} \frac{\sigma^{2}}{1-\alpha^{2}} u^{2}\right)
$$

satisfies

$$
\phi(u)=\gamma(u) \phi(\alpha u) \quad \text { for } u \in \mathbb{R} .
$$

Hence and from Theorem 3.1 we infer that

$$
\pi^{f}=N\left(\frac{m}{1-\alpha}, \frac{\sigma^{2}}{1-\alpha^{2}}\right) .
$$

Applying now Theorem 2.1 we see that Eq. (15) has a Lipschitz solution $\varphi$ : $\mathbb{R} \rightarrow Y$ if and only if

$$
\int_{\mathbb{R}} F(z) \exp \left(-\left(z-\frac{m}{1-\alpha}\right)^{2} / 2 \frac{\sigma^{2}}{1-\alpha^{2}}\right) d z=0 .
$$

In particular (cf., e.g., [5, pp. 299-300]):

2.1. If $\alpha \in(-1,1)$ and $\xi: \Omega \rightarrow \mathbb{R}$ is a random variable with the Gaussian law $N\left(m, \sigma^{2}\right)$, then the equation

$$
\varphi(x)=\int_{\Omega} \varphi(\alpha x+\xi(\omega)) P(d \omega)+x
$$

has a Lipschitz solution $\varphi: \mathbb{R} \rightarrow \mathbb{R}$ if and only if $m=0$. 
2.2. If $\alpha \in(-1,1), \xi: \Omega \rightarrow \mathbb{R}$ is a random variable with the standard Gaussian law $N(0,1), n \in \mathbb{N}$ and $\alpha_{0}, \alpha_{1}, \ldots, \alpha_{n} \in \mathbb{R}$, then the equation

$$
\varphi(x)=\int_{\Omega} \varphi(\alpha x+\xi(\omega)) P(d \omega)+\sum_{k=0}^{n} \alpha_{k} x^{k}
$$

has a Lipschitz solution $\varphi: \mathbb{R} \rightarrow \mathbb{R}$ if and only if

$$
\sum_{k=0}^{[n / 2]} \alpha_{2 k} \frac{(2 k) !}{k ! 2^{k}\left(1-\alpha^{2}\right)^{k}}=0 .
$$

3. Let $X$ be a real separable Hilbert space. Following [4] denote by $L_{1}^{+}(X)$ the set of all linear, symmetric and positive self-mappings of $X$ with finite trace.

Given a linear and symmetric $\Lambda: X \rightarrow X$ with $\|\Lambda\|<1$ and a Lipschitz mapping $F$ of $X$ into a separable Banach space $Y$ consider the equation

$$
\varphi(x)=\int_{\Omega} \varphi(\Lambda x+\xi(\omega)) P(d \omega)+F(x)
$$

assuming now that $\xi: \Omega \rightarrow X$ is a random variable with the Gaussian law $N(m, Q)$, where $m \in X$ and $Q \in L_{1}^{+}(X)$, and

$$
\Lambda Q=Q \Lambda \text {. }
$$

In this case

$$
f(x, \omega)=\Lambda x+\xi(\omega) \text { for }(x, \omega) \in X \times \Omega
$$

and (see [4, Sec. 1.2])

$$
\gamma(u)=\exp \left(i(m \mid u)-\frac{1}{2}(Q u \mid u)\right) \quad \text { for } u \in X .
$$

Put

$$
A=\left(I-\Lambda^{2}\right)^{-1} Q
$$

Since

$$
\left(I-\Lambda^{2}\right)^{-1}=\sum_{n=0}^{\infty} \Lambda^{2 n}
$$

the operator $\left(I-\Lambda^{2}\right)^{-1}$ is symmetric, and by (17) we have

$$
\left(I-\Lambda^{2}\right)^{-1} Q=Q\left(I-\Lambda^{2}\right)^{-1} \text { and } \Lambda A=A \Lambda .
$$

Consequently $A$ is symmetric and positive. As $Q$ has finite trace, so has $A$ and $A \in L_{1}^{+}(X)$. Moreover, the function $\phi: X \rightarrow \mathbb{C}$ given by 


$$
\phi(u)=\exp \left(i\left((I-\Lambda)^{-1} m \mid u\right)-\frac{1}{2}(A u \mid u)\right)
$$

satisfies

$$
\phi(u)=\gamma(u) \phi(\Lambda u) \quad \text { for } u \in X .
$$

Hence and from Theorem 3.1 and [4, Sec. 1.2] we infer that

$$
\pi^{f}=N\left((I-\Lambda)^{-1} m,\left(I-\Lambda^{2}\right)^{-1} Q\right) .
$$

Applying now Theorem 2.1 we see that Eq. (16) has a Lipschitz solution $\varphi$ : $X \rightarrow Y$ if and only if

$$
\int_{X} F d N\left((I-\Lambda)^{-1} m,\left(I-\Lambda^{2}\right)^{-1} Q\right)=0 .
$$

In particular, if $\Lambda: X \rightarrow X$ is linear and symmetric with $\|\Lambda\|<1$ and $\xi: \Omega \rightarrow X$ is a random variable with the Gaussian law $N(m, Q)$, where $m \in$ $X, Q \in L_{1}^{+}(X)$ and (17) holds, then the equation

$$
\varphi(x)=\int_{\Omega} \varphi(\Lambda x+\xi(\omega)) P(d \omega)+x
$$

has a Lipschitz solution $\varphi: X \rightarrow X$ if and only if $m=0$.

\section{Acknowledgements}

I thank Professor Gregory Derfel for calling my attention to the paper by O. K. Zakusilo.

This research was supported by the University of Silesia Mathematics Department (Iterative Functional Equations and Real Analysis program).

Open Access. This article is distributed under the terms of the Creative Commons Attribution 4.0 International License (http://creativecommons.org/licenses/by/4.0/), which permits unrestricted use, distribution, and reproduction in any medium, provided you give appropriate credit to the original author(s) and the source, provide a link to the Creative Commons license, and indicate if changes were made.

Publisher's Note Springer Nature remains neutral with regard to jurisdictional claims in published maps and institutional affiliations.

\section{References}

[1] Baron, K.: On the convergence in law of iterates of random-valued functions. Aust. J. Math. Anal. Appl. 6(1), Art. 3, 9 pp. (2009)

[2] Baron, K.: Weak law of large numbers for iterates of random-valued functions. Aequ. Math. 93, 415-423 (2019)

[3] Baron, K., Morawiec, J.: Lipschitzian solutions to linear iterative equations revisited. Aequ. Math. 91, 161-167 (2017) 
[4] Da Prato, G.: Introduction to stochastic analysis and Malliavin calculus. Appunti. Scuola Normale Superiore di Pisa (Nuova Serie), vol 13. Edizioni della Normale, Pisa (2014)

[5] Dudley, R.M.: Real Analysis and Probability. Cambridge Studies in Advanced Mathematics, vol. 74. Cambridge Univerity Press, Cambridge (2002)

[6] Kapica, R.: Convergence of sequences of iterates of random-valued vector functions. Colloq. Math. 97, 1-6 (2003)

[7] Kapica, R.: The geometric rate of convergence of random iteration in the Hutchinson distance. Aequ. Math. 93, 149-160 (2019)

[8] Kuczma, M., Choczewski, B., Ger, R.: Iterative Functional Equations. Encyclopedia of Mathematics and Its Applications, vol. 32. Cambridge University Press, Cambridge (1990)

[9] Parthasarathy, K.R.: Probability Measures on Metric Spaces. Probability and Mathematical Statistics, vol. 3. Academic Press, Inc., New York (1967)

[10] Zakusilo, O.K.: Some properties of random vectors of the form $\sum_{n=0}^{\infty} A^{i} \xi_{i}$. Teor. Verojatnost. i Mat. Statist. (Russian) 13, 59-62 (1975)

\section{Karol Baron}

Instytut Matematyki

Uniwersytet Śląski

Bankowa 14

40-007 Katowice

Poland

e-mail: baron@us.edu.pl

Received: January 26, 2019

Revised: March 26, 2019 